\title{
OCIO CULTURAL JUVENIL, INDICADOR SUBJETIVO DEL DESARROLLO HUMANO***
}

\section{YOUTH'S CULTURAL LEISURE, A SUBJECTIVE INDICATOR OF HUMAN DEVELOPMENT}

EVA SANZ ARAZURI

Universidad de la Rioja

Ma ÁNGeles VALDEMOROS SAN

EMETERIO

Universidad de la Rioja
CARMEN DUQue PALACIOS, Gobierno de La Rioja

FRANCISCO JAVIER GARCÍA-CASTILLA UNED

Recibido: $24 / 12 / 2018$

Aceptado: 24/04/2019

\section{RESUMEN}

El ocio cultural reporta numerosos beneficios que contribuyen al crecimiento personal en sus distintas dimensiones. El objetivo de este estudio es identificar la prácti-

\footnotetext{
* El texto que presentamos se vincula al Proyecto de Investigación «De los tiempos educativos a los tiempos sociales: la cotidianidad familiar en la construcción del ocio físico-deportivo juvenil» (EDU2012-39080-C07-05), y configura los inicios del proyecto de I+D+I «Ocio y bienestar en clave intergeneracional: de la cotidianidad familiar a la innovación social en las redes abuelos-nietos» (EDU2017-85642-R); ambos cofinanciados en el marco del Plan Nacional I+D+I con cargo a dos ayudas del Ministerio de Economía y Competitividad, y por el Fondo Europeo de Desarrollo Regional (FEDER).

A lo largo del documento se intentará utilizar un lenguaje inclusivo, si bien "en aplicación de la Ley $3 / 2007$, de 22 de marzo, para la igualdad efectiva de mujeres y hombres, toda referencia a cargos, personas o colectivos incluida en este documento en masculino, se entenderá que incluye tanto a mujeres como a hombres".
} 
ca de ocio cultural de la juventud como un indicador subjetivo del desarrollo humano, mediante el análisis de la percepción que el joven tiene sobre el influjo que estas experiencias ejercen en su dimensión física, psicológica, cognitiva y social. Se aplicó un cuestionario elaborado ad hoc a una muestra de 1023 jóvenes españoles. Las actividades culturales de ocio son importantes para la juventud, quien percibe que su práctica favorece su desarrollo humano, fundamentalmente en los aspectos psicológicos, emocionales, creativos y cognitivos.

Palabras clave: Cultura, Ocio, Beneficios, Subjetividad, Desarrollo Humano.

\section{ABSTRACT}

Cultural leisure provides numerous benefits that contribute to personal growth in its various dimensions. The goal of this study is to identify the practice of youth's cultural leisure as a subjective indicator of human development, through the analysis of young people's perception of the influence of such experiences on their physical, psychological, cognitive and social dimensions. An ad hoc questionnaire was administered to a sample of 1023 young Spaniards. Cultural leisure activities are important for youth, who perceive that its practice favors human development, primarily the psychological, emotional, creative and cognitive aspects.

Keywords: Culture, Leisure, Benefits, Subjectivity, Human Development

\section{INTRODUCCIÓN}

El ocio es uno de los valores más respetados por la ciudadanía y un derecho en sí mismo, especialmente valorado por la juventud (Arastegui y Silvestre, 2012; López Ruiz, 2011) constituyendo un importante pilar de desarrollo para el siglo XXI, que ocupa un espacio privilegiado en la era del conocimiento. Se trata de un requisito indispensable para la calidad de vida, llegando a convertirse en una necesidad, pues es entendido como una experiencia integral de la persona $\mathrm{y}$ un derecho humano fundamental que favorece el desarrollo personal y social (Cuenca, 2004, 2009; Cuenca y Goytia, 2012).

El ocio cultural responde a una experiencia libre, susceptible de ofrecer bienestar personal y conocimiento reflexivo, cuya vivencia se realiza mediante actividades con capital cultural implícito, caso de prácticas vinculadas con expresiones artísticas escénicas (cinematográficas, teatrales, pictóricas), audiovisuales, musicales, literarias, teatrales o museísticas (Valdemoros, Ponce-deLeón, Sanz y Tierno, 2015), que ponen en relación las demandas e intereses de 
las personas con los bienes culturales que la sociedad quiere preservar. Además de reactivar el interés por la cultura, posibilita la valoración y apreciación de las obras y ofrece oportunidades para el intercambio cultural (Orduna y Urp, 2010).

Esta relación entre libertad, creatividad cultural y bienestar social genera actividades diferenciadas entre expresivas y receptivas, en función de la manera de vivenciarlas; como ejemplo, en el caso de la música, ésta puede ser experimentada de manera expresiva, cuando se toca un instrumento o se crea una composición musical; y de manera receptiva cuando se asiste a un concierto (Valdemoros et al., Op. cit.). La literatura científica constata que la práctica de actividades de ocio cultural posee múltiples beneficios, tanto específicos de cada actividad como comunes a todas ellas, que contribuyen al desarrollo integral del individuo (Bygren et al., 2009; De Vries \& De Graaf, 2008; Grossi et al., 2011; Pinxten \& Lievens, 2014; Veenstra \& Patterson, 2012). Algunas bondades se vinculan con aspectos psicológicos y emocionales, como la mejora de la capacidad para expresar e interpretar emociones (Bygren et al., Op. cit.) y la expresión de la diversidad cultural (Katz-Gerro, Raz, \& Yaish, 2009), lo que favorece el desarrollo de habilidades de comunicación y para la vida, el pensamiento crítico y analítico, así como la autorregulación (García-Castilla, De-Juanas, y LópezNoguero, 2016).

Otros beneficios del ocio cultural se asocian con la mejora de la salud en su concepción holista (Cuypers et al., 2012), optimizando, concretamente, la salud mental (Pinxten \& Lievens, Op. cit.) y aumentando la capacidad para expresarse e interpretar emociones (Bygren et al., Op. cit.). Incluso, algunos autores (Cassián, 2012) declaran que la práctica de actividades culturales es una necesidad básica para la salud mental de las personas.

No pueden obviarse los beneficios que el ocio cultural comporta en la esfera social por su influjo positivo en la movilidad social (McCammon et al., 2012), por su significado simbólico (Elchardus, 2009), por favorecer valores como el respeto, el compañerismo, el trabajo en equipo, la cohesión social y la cooperación (Ramírez-Marrero et al., 2004); así como por beneficiar la interacción familiar y el desarrollo cultural, social e individual de sus miembros (Magaña, 2006).

También, el ocio cultural integra virtudes en relación con el desarrollo creativo-cognitivo de la persona, ya que se confirma que su práctica favorece la creatividad (Cuenca, 2000) e influye favorablemente en el rendimiento y los resultados académicos (De Vries \& De Graaf, Op. cit.), lo que mejora de un modo concreto la comprensión lectora y la habilidad matemática (Mads, 2009). 
Apenas existen estudios que relacionan estas actividades con el desarrollo físico de la persona, descubriéndose, tan solo, el estudio de Conejo (2012) que verifica que la práctica de ocio cultural favorece el desarrollo psicomotor.

Hasta ahora se han puesto de manifiesto las bondades del ocio cultural sin diferenciar ninguna tipología de actividad. Si bien, estudios previos distinguen unos $\mathrm{u}$ otros beneficios en función de la naturaleza de la actividad realizada.

Así, la práctica de actividades como la lectura, ver películas e ir al teatro se asocia a una mejora de la cognición, dado que mediante ellas se optimiza la fluidez y la comprensión. Incluso contribuye a la prevención de dificultades de aprendizaje y problemas de comportamiento (Axelsson, Granlund, \& Wilder, 2013; Moreno, 2013), gracias a la transmisión de contenidos, emociones, valores y modelos de comportamiento que estas prácticas comportan (Moreno, 2002).

Más en concreto, la práctica de la lectura viabiliza el desarrollo y enriquecimiento intelectual (Díaz, 2009; Gil, 2011), lingüístico (Biemiller \& Boote, 2006) y de la comprensión (Piasta et al., 2012). También, mejora el rendimiento académico y las calificaciones escolares (Cromley, 2009; Díaz Op. cit.) pues permite evolucionar el vocabulario receptivo y expresivo, la comprensión lectora y el lenguaje (Conlon et al., 2006; Zucker et al., 2013), además de estimular el desarrollo neuropsicoafectivo y social, lo que favorece el sentido crítico y las habilidades para la vida. Todo ello, impulsa la educación en valores, así como apreciar nuestro patrimonio cultural (Álvarez y García, 2013; Aristizábal et al., 2013; Cerrillo, Yubero y Larrañaga, 2004; Presentado, 2014). En definitiva, el hábito lector beneficia el desarrollo integral del individuo (Yubero y Larrañaga, 2010), por lo que la lectura debe ser considerada un indicador cardinal del desarrollo humano tal y como señala la Organización para la Cooperación y el Desarrollo Económicos (OCDE).

Las actividades musicales favorecen el desarrollo de actitudes creativas, de pensamiento relacional, de respeto y apertura (Moreno, 2011) y fomentan el crecimiento personal, intelectual, psicomotor y socioafectivo del individuo al desarrollar la atención, la memorización, la concentración, la agilidad mental y el desarrollo de la creatividad. Además, produce una sensación de placer y felicidad en las personas (Conejo et al., Op. cit); cultiva, asimismo, nuestra inteligencia emocional, lo que favorece la ampliación de nuestro conocimiento y capacidad moral, el pensamiento relacional, así como el desarrollo de actitudes de respeto y apertura (López Quintás, 2013; Madariaga y Cuenca, 2011).

Las visitas a museos o exposiciones constituyen una oportunidad para el encuentro entre generaciones y culturas, dado que posibilita la creación de espa- 
cios para el aprendizaje y la acción didáctica; ya sea en entidad real o en red, como es el caso de los museos pedagógicos que se encuentran en Internet (Álvarez y García, 2013).

Otras actividades artísticas, como por ejemplo escribir, pintar, esculpir, componer música o tocar un instrumento, promueven la creatividad y el espíritu crítico (Caride, Lorenzo, y Rodríguez, 2012), favorecen la movilidad social y desarrollan competencias culturales que fomentan la participación en arte y cultura, lo que ofrece oportunidades para el desarrollo de la creatividad y la innovación (Vanherwegen \& Lievens, 2014).

La revisión bibliográfica de la literatura científica y la investigación más reciente constatan que los jóvenes se decantan por actividades culturales relacionadas con escuchar música, ir al cine y ver la TV (Ballesteros et al., 2009; Caba1lo, Caride, y Gradaille, 2012; López Ruiz, 2011; Moreno y Rodríguez, 2014; Muñoz y Olmos, 2010).

Datos del Ministerio de Educación, Cultura y Deporte (MECD) de 2015 señalan una tendencia a la mayor participación de los jóvenes en todas las actividades culturales, en general. Si bien, se trata de hallazgos que se contradicen con los de otros autores quienes inciden en un notable descenso, en el caso de las visitas a museos (López Ruiz, 2011; Moreno y Rodríguez, Op. cit.), de los hábitos lectores (Berntsson \& Ringsberg, 2014) y la asistencia a espectáculos deportivos (López Ruiz, Op. cit.; Moreno y Rodríguez, Op. cit.). Sin embargo, coinciden en la consideración de que ver la televisión se constituye en la actividad preferida por los jóvenes (Caballo, Caride, y Gradaille, Op. cit.).

Finalmente, se detectan investigaciones que corroboran el bajo porcentaje de jóvenes que tocan un instrumento musical, realizan composiciones literarias y/o dibujan (López Ruiz, Op. cit.; Moreno y Rodríguez, Op. cit.).

En definitiva, son numerosos los estudios que analizan el potencial del ocio cultural para el desarrollo humano, en su dimensión psicológica, creativa, cognitiva y social. No obstante, aquellos que estudian la percepción que tienen los jóvenes acerca de cómo la práctica de ocio cultural es susceptible de influir en su calidad de vida y el desarrollo humano apenas cuentan con representación en el ámbito científico.

Se plantea como objetivo del presente artículo identificar la práctica de ocio cultural de la juventud como un indicador subjetivo del desarrollo humano, mediante el análisis de la percepción que el joven tiene sobre el influjo que estas experiencias ejercen en su crecimiento integral, teniendo en cuenta variables de 
género y prestando especial atención a las tres prácticas de ocio cultural que sitúan como preferentes.

En este sentido, la evidencia científica se erige como base sólida que ha de orientar las nuevas políticas sociales que refuercen y consoliden, desde la juventud, condiciones personales y sociales para favorecer un ocio cultural que optimice la calidad de vida de las personas.

Las Administraciones Públicas tienen la responsabilidad de generar contextos socioeconómicos y culturales favorables para promoción del ocio, como un derecho universalmente reconocido $\mathrm{y}$, más en concreto, de la práctica de ocio cultural, mediante la articulación de diferentes políticas públicas de ámbito estatal que incidan en este objetivo.

De ahí la importancia de identificar cuáles son las actividades de ocio cultural más relevantes para los jóvenes españoles, detectando sus preferencias y la percepción que tienen acerca de su influjo para el desarrollo emocional, cognitivo, creativo y social; lo que viabilizará la obtención de claves y medidas de actuación que incrementen los tiempos de ocio cultural juvenil y contribuyan, de este modo, al aumento de los beneficios que estas experiencias aportan al individuo.

\section{MÉTODO}

\section{PARTICIPANTES}

En el presente estudio, de tipo cuantitativo y ex post facto, participaron 1023 estudiantes españoles de Bachillerato de edades comprendidas entre los 15 y los 19 años. El 54,5\% eran mujeres $(n=558)$ y el $45,5 \%$ hombres $(n=465)$. La selección de los participantes se efectuó a través de un muestreo aleatorio simple de la población, consiguiendo representación proporcional de todas las áreas geográficas del territorio español.

\section{INSTRUMENTOS}

El tipo de instrumento elegido para recoger información sobre las variables del estudio fue el cuestionario, siendo definidas las siguientes variables:

- Género: variable dicotómica que identifica si el encuestado es hombre o mujer.

- Se consideraron las actividades culturales como actividades de ocio prioritarias: se trata de una variable dicotómica [sí /no] que recoge si el 
joven identifica alguna actividad cultural entre sus tres preferencias para dedicar su tiempo de ocio. Se tuvieron en cuenta las actividades culturales, a saber: escuchar música, ver películas en el cine o en casa, leer libros que le gustan, ir al teatro, ir a museos, galerías o exposiciones, ir a conciertos o festivales, practicar alguna actividad artística, musical o literaria.

- Número de actividades culturales prioritarias: variable numérica, con recorrido posible desde 0 hasta 3 , que identifica el número de actividades culturales que se encuentran entre los tres ocios prioritarios del joven.

- Grado en que los jóvenes perciben que la práctica de ocio cultural les aporta:

- Una mejora en la forma física

- Un bienestar psicológico

- Un desarrollo cognitivo-creativo

- Una mejora de las destrezas manuales

- Una mejora de las relaciones sociales

Estas cinco variables son ordinales y registradas a través de una escala tipo likert de 5 puntos, donde 1 indica nada, 2 significa poco, 3 algo, 4 bastante y 5 mucho.

\section{PROCEDIMIENTO}

El instrumento fue aplicado a todos los estudiantes de los grupos-clase concretos de centros educativos seleccionados aleatoriamente y distribuidos por todas las áreas geográficas españolas. Mediante un escrito formal se comunicó la intención del estudio y se solicitó la participación en el mismo de manera voluntaria a los responsables educativos de las distintas Comunidades Autonómicas, a los directores de los respectivos centros educativos, y a los padres y madres de los estudiantes. Se garantizó el anonimato, respeto y confidencialidad con el propósito de cumplir con los lineamientos que se encuentran en la Declaración de Helsinki (Asamblea Médica Mundial, 2008) en la que se establecen los principios éticos para las investigaciones con seres humanos. A su vez, durante y después de todo el proceso de investigación se procedió de acuerdo con las disposiciones de la Ley Orgánica Española 15/1999, de 13 de diciembre, para la protección de datos personales. 


\section{ANÁLISIS DE RESULTADOS}

Con el fin de conocer el porcentaje de alumnos de bachillerato que consideran las actividades culturales entre sus actividades prioritarias de ocio, se llevó a cabo un análisis descriptivo, mediante el estadístico de frecuencia. Seguidamente, centrando el estudio en el colectivo de estudiantes que consideran importantes las actividades culturales y con la intención de descubrir la percepción de estos sobre las implicaciones que estas actividades tienen para su desarrollo físico, psicológico, cognitivo y creativo y social, se utilizó la media y la desviación estandar.

En un nivel mayor de concreción, tratando de descubrir y describir si la percepción sobre la aportación de las actividades culturales en su desarrollo físico, psicológico, creativo-cognitivo y social muestra diferencias significativas atendiendo a las variables género, número de actividades culturales prioritarias y tipo de actividades culturales prioritarias, se llevan a cabo la prueba paramétrica $t$ de student para muestras independientes y la prueba de varianza (ANOVA) de un factor. Los supuestos de normalidad y homocedasticidad se acreditaron a través de la homogeneidad de las varianzas. Finalmente, se efectuaron contrastes mediante comparaciones múltiples post-hoc; y cuando el estadístico de Levene asumió varianzas iguales se empleó la prueba de Tukey, mientras que cuando las varianzas presentaban valores diferentes se recurría a la prueba de Games-Howell. El nivel de significatividad considerado en todo momento fue $\mathrm{p}<0,05$.

\section{RESULTADOS}

El 64,7\% de los estudiantes de Bachillerato del estado español identifican, al menos, una actividad cultural entre sus tres actividades de ocio más importantes. Un 18,2\% identifican una actividad cultural como su ocio principal, un $23,7 \%$ como su segundo ocio principal y un $22,8 \%$ como su tercer ocio principal.

La actividad de ocio cultural más importante señalada por estos jóvenes es escuchar música $(24,3 \%)$, seguido de las actividades artísticas (12,2\%), la lectura de libros $(11 \%)$, ver películas $(9,1 \%)$, asistir a espectáculos deportivos $(1 \%)$, ir a conciertos $(0,9 \%)$, ir al teatro $(0,4 \%)$ e ir a museos $(0,2 \%)$. 


\section{GRÁFICO 1}

Actividades culturales más practicadas de los estudiantes de bachillerato dentro de sus tres actividades de ocio más importantes

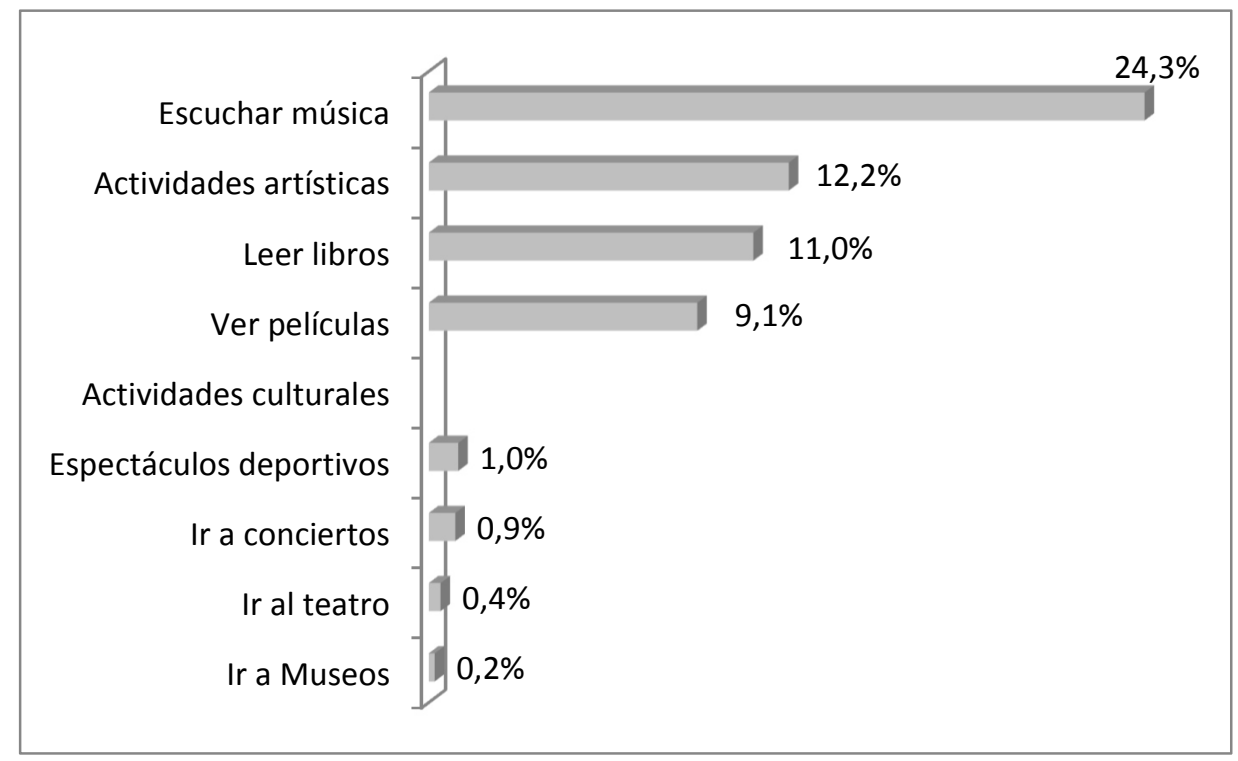

El análisis inferencial no detectó ningún tipo de asociación entre el género del adolescente y el hecho de señalar alguna actividad cultural entre sus tres experiencias de ocio más importantes $(\pi=-, 028 \mathrm{p}=, 237)$; y es que chicos y chicas señalan en similar proporción una experiencia de este tipo entre sus preferentes ( $55 \%$ y $57,8 \%$, respectivamente).

Centrando el estudio exclusivamente en el 64,7\% $(n=727)$ de los jóvenes que identifican al menos una actividad cultural entre sus tres actividades de ocio más importantes, se confirma que estos consideran que las actividades culturales que práctican les ayudan a desarrollarse en gran medida en su dimensión psicológica $(=4,37 \pm 0,883)$ y cognitiva-creativa $(=3,92 \pm 1,122)$, pero no mucho en el ámbito social $(=2,62 \pm 1,413)$ y menos en el físico $(=1,82 \pm 1,153$ en la mejora de la forma física y $=2,28 \pm 1,232$ en el desarrollo de nuevas destrezas manuales) (CUADRO 1). 


\section{CUADRO 1}

Estadísticos descriptivos: percepción sobre el desarrollo humano que aportan las actividades culturales

\section{Cuando practico actividades culturales...

$$
\bar{X} \pm \mathrm{ds}
$$

...estoy más en forma, controlo mejor mis movimientos, mantengo o $\quad 1,82 \pm 1,153$ mejoro mi condición física

...me siento más satisfecho/a, disfruto, me divierte

$4,37 \pm 0,883$

...soy más creativo/a, adquiero conocimientos, aprendo cosas y 3,92 $\pm 1,122$ amplio mi mundo

... desarrollo nuevas destrezas manuales y adquiero o perfecciono 2,02 $\pm 1,232$ habilidades técnicas

...hago cosas diferentes con más gente, me siento parte de un grupo, 2,62 $\pm 1,413$ me ayuda a relacionarme mejor con los demás

El análisis inferencial evidencia que el ocio cultural satisface y divierte por igual a mujeres y hombres $(\mathrm{p}=0,632)$; ambos colectivos coinciden en declarar que estas prácticas provocan bastante desarrollo de su creatividad y conocimientos $(\mathrm{p}=0,431)$; tanto ellos como ellas indican estar poco conformes respecto a la afirmación de que les ayuda a mejorar su forma física $(p=0,585)$ y las destrezas manuales $(\mathrm{p}=0,567)$ y entre poco y algo conformes con que les permiten impulsar sus relaciones sociales (CUADRO 2).

\section{CUADRO 2}

Resumen t de Student para muestras independientes: percepción sobre el desarrollo humano que aporta el ocio cultural en relación con el género del estudiante

\begin{tabular}{|c|c|c|c|c|c|c|c|}
\hline \multirow[t]{2}{*}{$\begin{array}{ll}\text { Cuando } & \text { practico } \\
\text { actividades culturales... }\end{array}$} & \multicolumn{2}{|c|}{$\begin{array}{l}\text { Prueba de } \\
\text { Levene para } \\
\text { la igualdad } \\
\text { de varianzas }\end{array}$} & \multicolumn{3}{|c|}{$\begin{array}{l}\text { Prueba t para la } \\
\text { igualdad de medias }\end{array}$} & \multirow[t]{2}{*}{$\bar{X} \pm \mathrm{ds}$} & \\
\hline & $\mathrm{F}$ & $p^{I}$ & $t$ & $\mathrm{gl}$ & $p$ & & \\
\hline $\begin{array}{l}\text {...estoy más en forma, } \\
\text { controlo mejor mis } \\
\text { movimientos, mantengo o } \\
\text { mejoro mi condición física }\end{array}$ & 1,161 & 282 &,- 546 & 403 & ,585 & $\begin{array}{l}\text { Mujer= } \\
1,101 \\
\text { Hombre= } \\
1,237\end{array}$ & $1,80 \pm$ \\
\hline $\begin{array}{l}\text {..me siento más } \\
\text { satisfecho/a, disfruto, me } \\
\text { divierte }\end{array}$ & 0,004 & ,948 & $-0,479$ & 427 & ,632 & $\begin{array}{l}\text { Mujer= } \\
0,873 \\
\text { Hombre }= \\
0,902\end{array}$ & $\begin{array}{r}4,35 \pm \\
4,40 \quad \pm\end{array}$ \\
\hline
\end{tabular}




\begin{tabular}{|c|c|c|c|c|c|c|c|}
\hline $\begin{array}{l}\text {...soy más creativo/a, } \\
\text { adquiero conocimientos, } \\
\text { aprendo cosas y amplio mi } \\
\text { mundo }\end{array}$ & 0,693 & 406 & $-0,789$ & 421 & ,431 & $\begin{array}{l}\text { Mujer= } \\
1,137 \\
\text { Hombre }= \\
1,096\end{array}$ & $3,89 \pm$ \\
\hline $\begin{array}{l}\ldots \text { desarrollo nuevas } \\
\text { destrezas manuales y } \\
\text { adquiero o perfecciono } \\
\text { habilidades técnicas }\end{array}$ & 0,453 & ,305 & $-0,894$ & 411 & ,567 & $\begin{array}{l}\text { Mujer= } \\
0,757 \\
\text { Hombre= } \\
0,934\end{array}$ & $\begin{array}{r}2,04 \pm \\
1,97 \quad \pm\end{array}$ \\
\hline $\begin{array}{l}\text {..hago cosas diferentes } \\
\text { con más gente, me siento } \\
\text { parte de un grupo, me } \\
\text { ayuda a relacionarme }\end{array}$ & 1,666 & , 198 & $-1,714$ & 410 & ,087 & $\begin{array}{l}\text { Mujer }= \\
1,371 \\
\text { Hombre }= \\
1,472\end{array}$ & $\begin{array}{r}2,52 \pm \\
2,77 \quad \pm\end{array}$ \\
\hline
\end{tabular}

${ }^{l}$ Prueba de Levene: $p>0.05$ en todos los casos luego se asume la igualdad de las varianzas.

Los resultados del ANOVA de un factor, para el número de actividades culturales señaladas por los estudiantes de Bachillerato entre las tres actividades de ocio más importantes, no muestran diferencias significativas ni en cuanto a la mejora de la forma física, ni en cuanto a la satisfacción, disfrute y diversión; ni a la creatividad, las destrezas manuales y la facilidad para establecer relaciones sociales que consideran que proporciona la práctica de actividades culturales de ocio (CUADRO 3).

\section{CUADRO 3}

Resumen ANOVA de un factor: percepción sobre el desarrollo humano que aportan las actividades culturales y número de actividades señaladas entre las tres más importantes

\begin{tabular}{|c|c|c|c|c|c|c|c|c|}
\hline 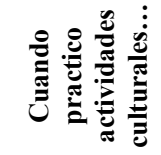 & 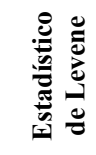 & 2 & & 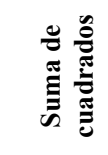 & 50 & 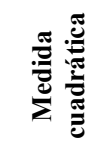 & 5 & $=$ \\
\hline \multirow{3}{*}{$\begin{array}{l}\text {...estoy más } \\
\text { en forma, } \\
\text { controlo } \\
\text { mejor mis } \\
\text { movimientos, } \\
\text { mantengo o } \\
\text { mejoro mi } \\
\text { condición } \\
\text { física }\end{array}$} & 3,118 & ,045 & Intergrupos & 2,982 & 2 & 1,491 & 1,121 &, 327 \\
\hline & & & Intragrupos & 534,571 & 402 & 1,330 & & \\
\hline & & & Total & 537,553 & 404 & & & \\
\hline \multirow{2}{*}{$\begin{array}{l}\text {...me siento } \\
\text { más } \\
\text { satisfecho/a, }\end{array}$} & 1,373 &, 254 & Intergrupos & 1,493 & 2 & 0,746 & 0,956 & ,385 \\
\hline & & & Intragrupos & 332,668 & 426 & 0,781 & & \\
\hline
\end{tabular}




\begin{tabular}{|c|c|c|c|c|c|c|c|c|}
\hline $\begin{array}{l}\text { disfruto, me } \\
\text { divierte }\end{array}$ & & & Total & 34,161 & 428 & & & \\
\hline \multirow{3}{*}{$\begin{array}{l}\text {...soy más } \\
\text { creativo/a, } \\
\text { adquiero } \\
\text { conocimien- } \\
\text { tos, aprendo } \\
\text { cosas y } \\
\text { amplio mi } \\
\text { mundo }\end{array}$} & 2,878 & ,057 & Intergrupos & 7,007 & 2 & 3,503 & 2,807 & ,062 \\
\hline & & & Intragrupos & 524,283 & 420 & 1,248 & & \\
\hline & & & Total & 531,290 & 422 & & & \\
\hline \multirow{3}{*}{$\begin{array}{l}\ldots \text { desarrollo } \\
\text { nuevas } \\
\text { destrezas } \\
\text { manuales y } \\
\text { adquiero o } \\
\text { perfecciono } \\
\text { habilidades } \\
\text { técnicas }\end{array}$} & 1,949 & ,000 & Intergrupos & 58,865 & 2 & 7,959 & 3,588 & ,202 \\
\hline & & & Intragrupos & 336,746 & 506 & 3,329 & & \\
\hline & & & Total & 595,610 & 609 & & & \\
\hline \multirow{3}{*}{$\begin{array}{l}\text {...hago cosas } \\
\text { diferentes con } \\
\text { más gente, } \\
\text { me siento } \\
\text { parte de un } \\
\text { grupo, me } \\
\text { ayuda a } \\
\text { relacionarme }\end{array}$} & 3,823 & ,023 & Intergrupos & 1,511 & 2 & 0,756 & 0,377 & ,686 \\
\hline & & & Intragrupos & 819,600 & 409 & 2,004 & & \\
\hline & & & Total & 821,111 & 411 & & & \\
\hline
\end{tabular}

Centrando el análisis inferencial en el tipo de actividades culturales señaladas por los estudiantes de Bachillerato entre las tres actividades de ocio más importantes, tampoco se detectan diferencias significativas ni en cuanto al desarrollo de la forma física, ni a la satisfacción, disfrute y diversión; la creatividad o a la facilidad para establecer relaciones sociales que consideran que proporciona la práctica de actividades culturales de ocio. Sin embargo, sí se revelan diferencias significativas en cuanto a la mejora de las destrezas manuales $(\mathrm{p}=, 002)(\mathrm{CUADRO} 4)$. 


\section{CUADRO 4}

Resumen ANOVA de un factor: percepción sobre el desarrollo humano que aporta las actividades culturales y tipo de actividades señaladas entre las tres más importantes

\begin{tabular}{|c|c|c|c|c|c|c|c|c|}
\hline 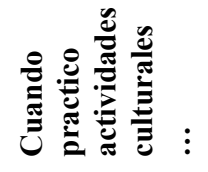 & 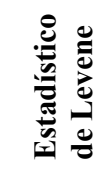 & 2 & & 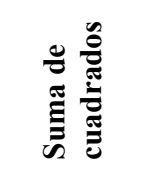 & 50 & 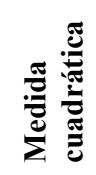 & 工 & 2 \\
\hline \multirow{3}{*}{$\begin{array}{l}\text {...estoy más } \\
\text { en forma, } \\
\text { controlo mejor } \\
\text { mis } \\
\text { movimientos, } \\
\text { mantengo o } \\
\text { mejoro mi } \\
\text { condición } \\
\text { física }\end{array}$} & 4,758 &, 000 & Intergrupos & 18,993 & 6 & 3,165 & 2,295 &, 056 \\
\hline & & & Intragrupos & 335,151 & 243 & 1,379 & & \\
\hline & & & Total & 354,144 & 249 & & & \\
\hline \multirow{3}{*}{$\begin{array}{l}\text {...me siento } \\
\text { más } \\
\text { satisfecho/a, } \\
\text { disfruto, me } \\
\text { divierte }\end{array}$} & 2,284 & ,036 & Intergrupos & 34,641 & 6 & 5,773 & 1,230 & ,291 \\
\hline & & & Intragrupos & 1206,814 & 257 & 4,696 & & \\
\hline & & & Total & 1241,455 & 263 & & & \\
\hline \multirow{3}{*}{$\begin{array}{l}\text {...soy más } \\
\text { creativo/a, } \\
\text { adquiero } \\
\text { conocimientos, } \\
\text { aprendo cosas } \\
\text { y amplio mi } \\
\text { mundo }\end{array}$} & 4,111 & ,001 & Intergrupos & 47,004 & 6 & 7,834 & 1,912 & ,079 \\
\hline & & & Intragrupos & 1024,062 & 250 & 4,096 & & \\
\hline & & & Total & 1071,066 & 256 & & & \\
\hline \multirow{3}{*}{$\begin{array}{l}\text {... desarrollo } \\
\text { nuevas } \\
\text { destrezas } \\
\text { manuales y } \\
\text { adquiero o } \\
\text { perfecciono } \\
\text { habilidades } \\
\text { técnicas }\end{array}$} & 6,499 & ,000 & Intergrupos & 47,754 & 6 & 7,959 & 3,588 &, $002 *$ \\
\hline & & & Intragrupos & 547,857 & 247 & 2,218 & & \\
\hline & & & Total & 595,610 & 253 & & & \\
\hline \multirow{3}{*}{$\begin{array}{l}\text {...hago cosas } \\
\text { diferentes con } \\
\text { más gente, me } \\
\text { siento parte de } \\
\text { un grupo, me } \\
\text { ayuda a } \\
\text { relacionarme }\end{array}$} & 1,110 & ,357 & Intergrupos & 24,140 & 6 & 4,023 & 2,132 &, 051 \\
\hline & & & Intragrupos & 464,196 & 246 & 1,887 & & \\
\hline & & & Total & 488,337 & 252 & & & \\
\hline
\end{tabular}


Mediante comparaciones múltiples se realiza la prueba de Games-Howell, se detecta que los estudiantes que indican las actividades artísticas entre sus tres actividades de ocio más importantes $(=2,000)$ perciben, en mayor medida que aquellos que identifican las visitas a museos como actividad de ocio prioriaria $(=0,200)$, que el ocio cultural les proporciona mejoras de habilidades y destrezas manuales $(\mathrm{p}=, 002)$. Sin embargo, se observa que no existen diferencias significativas entre el resto de las actividades culturales (CUADRO 5).

\section{CUADRO 5}

Comparaciones múltiples mediante Games Howell: análisis de la percepción sobre el desarrollo de destrezas manuales que aporta el ocio cultural y el tipo de actividades señaladas entre las tres más importantes

\begin{tabular}{|c|c|c|c|c|}
\hline $\begin{array}{l}\text { (I) Tipo de OC entre } \\
\text { los } 3 \text { ocios más } \\
\text { importantes }\end{array}$ & $\begin{array}{l}\text { (J) Tipo de OC } \\
\text { entre los } 3 \\
\text { ocios más } \\
\text { importantes }\end{array}$ & $\begin{array}{l}\text { Diferencia de } \\
\text { medias (I-J) }\end{array}$ & Error típico & $p$ \\
\hline \multirow{6}{*}{ Escuchar música } & Ver películas &, 13008 & ,28886 & 999 \\
\hline & Leer libros &,- 19963 & 35435 & ,997 \\
\hline & Ir al teatro &, 11341 & 60933 & 1,000 \\
\hline & Ir a museos &, 51341 & 22645 &, 373 \\
\hline & Ir a conciertos & ,38008 & ,34984 & 895 \\
\hline & Act. artísticas & $-1,28659$ & ,41497 &, 054 \\
\hline \multirow{5}{*}{ Ver películas } & Leer libros &,- 32971 & ,43179 & ,987 \\
\hline & Ir al teatro &,- 01667 & 65739 & 1,000 \\
\hline & Ir a museos & ,38333 & 33491 &, 373 \\
\hline & Ir a conciertos &, 25000 & ,42810 &, 895 \\
\hline & Act. artísticas & $-1,41667$ & ,48279 &, 054 \\
\hline \multirow{4}{*}{ Leer libros } & Ir al teatro &, 31304 & ,68868 & ,999 \\
\hline & Ir a museos &, 71304 & 39279 &, 551 \\
\hline & Ir a conciertos &, 57971 & , 47475 &, 867 \\
\hline & Act. artísticas & $-1,08696$ &, 52460 &, 384 \\
\hline \multirow{3}{*}{ Ir al teatro } & Ir a museos &, 40000 & ,63246 & ,992 \\
\hline & Ir a conciertos & ,26667 & 68638 & ,999 \\
\hline & Act. Artísticas & $-1,40000$ &, 72175 &, 508 \\
\hline \multirow{2}{*}{ Ir a museos } & Ir a conciertos &,- 13333 & ,38873 & 1,000 \\
\hline & Act. artísticas & $-1,80000 *$ &, 44824 &, $006 *$ \\
\hline Ir a conciertos & Act. artísticas & $-1,66667$ & ,51157 & ,091 \\
\hline
\end{tabular}

$P<, 005$ 


\section{CONCLUSIONES}

Puede afirmarse que el colectivo juvenil concede mucha importancia a las actividades culturales en su tiempo de ocio y que estas experiencias se constituyen como un indicador subjetivo de su desarrollo personal. Sobre todo, en su dimensión psicológica, emocional, cognitiva y creativa, dado que perciben que estos hábitos contribuyen, en estas esferas, a su crecimiento humano.

Las actividades de ocio cultural señaladas como más importantes para los jóvenes objeto de este estudio son: escuchar música, realizar actividades artísticas, leer libros y ver películas. Sin embargo, los jóvenes declaran conceder escasa importancia a otras prácticas de ocio cultural como la asistencia a espectáculos deportivos, a conciertos, al teatro y a museos.

Los jóvenes identifican la práctica de actividades culturales como un indicador subjetivo de desarrollo humano, dado que perciben que dichas experiencias les ayudan a desarrollarse como personas. Fundamentalmente, en su dimensión psicológica-emocional y cognitiva-creativa, sin descubrirse diferencias significativas según el sexo, el número de actividades culturales señaladas entre las tres principales de ocio o el tipo de actividad.

Dicha percepción puede deberse, por un lado, al potencial psicológico del ocio cultural, constatado por la literatura científica, para la mejora de la gestión de las emociones, de la expresión de la diversidad cultural, del desarrollo de habilidades de comunicación y para la vida, de la autorregulación y del pensamiento crítico y analítico. Por otro lado, a la capacidad demostrada de los hábitos culturales para favorecer la creatividad, el rendimiento y los resultados académicos, debido a que mejora tanto la comprensión lectora como la habilidad matemática.

En otro orden, el colectivo juvenil al que va dirigido este estudio está de acuerdo con las conclusiones de investigaciones previas en que las actividades culturales también influyen en su desarrollo social, dado que sus percepciones subjetivas indican un desarrollo inferior al de las dimensiones psicológicaemocional y cognitiva-creativa. Y mucho menos perciben que estos hábitos favorezcan a su dimensión física, lo que sintoniza con la literatura científica examinada.

Esta percepción, aun siendo un elemento subjetivo, coincide con la realidad científicamente constatada de que actividades como la lectura o ver películas se asocian a una mejora de la cognición, lo que posibilita el enriquecimiento intelectual, lingüístico y de la comprensión. Además, estimula el desarrollo 
neuropsicoafectivo, la educación en valores y el aprecio de nuestro patrimonio cultural; cuestiones que, por ende, benefician el desarrollo integral del individuo.

También las actividades artísticas, como escribir, pintar, esculpir, componer música o tocar un instrumento, señaladas como preferentes entre nuestros jóvenes, promueven la creatividad y el espíritu crítico, desarrollan competencias culturales y actitudes creativas e innovadoras. Todo ello, refuerza la percepción de nuestros jóvenes de que las actividades culturales contribuyen a su desarrollo humano y, más en concreto, en su dimensión cognitiva-creativa. Se destaca que los jóvenes que indican las actividades artísticas entre sus tres actividades de ocio más importantes perciben, en mayor medida que aquellos que identifican las visitas a museos como actividad prioriaria, que el ocio cultural les proporciona mejora en las habilidades y destrezas manuales. Sin embargo, resulta interesante destacar que no perciben que las actividades artísticas les proporcionen mayor desarrollo de las habilidades y destrezas manuales que las actividades culturales relacionadas con escuchar música, ver películas en el cine o en casa, leer libros que le gustan, ir al teatro, ir a conciertos o festivales.

Puede concluirse que para el conjunto de jóvenes que forman parte de este estudio son muy importantes las actividades culturales en su tiempo de ocio y perciben que su práctica favorece su crecimiento como personas. Fundamentalmente en los aspectos psicológicos, emocionales, creativos y cognitivos, por lo que se considera de gran interés que las políticas sociales intensifiquen sus esfuerzos en ofrecer oportunidades de ocio cultural desde unas condiciones facilitadoras y estimuladoras que consoliden estas alternativas creativas, artísticas e intelectuales entre los ocios prioritarios de los jóvenes. Asimismo, se constata la necesidad de continuar esta línea de investigación, dado que se evidencia que el ocio cultural de la juventud es un indicador subjetivo de su desarrollo humano.

Por último, se culmina este artículo con unas propuestas para la reflexión y la acción que las políticas de ocio y juventud de las instituciones implicadas tienen el deber de generar, y que se materializan en:

- Promocionar actividades de ocio cultural de forma integradora con aquellos sectores de la población que por sus condiciones socioeconómicas tienen más dificultad para su acceso, facilitando la participación activa.

- Hacer partícipes y beneficiarios a todos los colectivos en aquellos proyectos y actividades que tienden a la promoción y protección del ocio y la cultura, favoreciendo la práctica del colectivo juvenil como aspecto primordial. 
- Integrar el ocio cultural en un mercado cada vez más abierto y digitalizado.

- Centrar esfuerzos en ofrecer espacios y materiales a los jóvenes para realizar actividades culturales que fomentan el aprendizaje y el desarrollo integral de la persona.

- Configurar un entorno socioeconómico y cultural propicio para la práctica de ocio cultural. Para lo que se debe priorizar a la juventud en la agenda política y social, tanto en el ámbito nacional como regional y local, favoreciendo un diálogo continuo entre los diferentes agentes implicados.

- Hacer partícipes a todas las administraciones, al tejido social y empresarial, así como, a la sociedad civil, reconociendo la necesidad de empoderar y fortalecer a la juventud mediante el ocio cultural, y movilizando todos los recursos necesarios para asegurar un desarrollo humano justo y de calidad.

\section{REFERENCIAS BIBLIOGRÁFICAS}

Álvarez, C. y García, M. (2013). Escuela, museo, familia, lectura y valores: Proyecto de educación en valores para la Red de Museos Etnográficos de Asturias. Íber. Didáctica de las Ciencias Sociales, Geografia e Historia, 73(1), 73-80.

Arastegui, I. y Silvestre, M. (2012). El ocio como valor en la sociedad actual. Arbor. Ciencia, pensamiento y cultura, 188(754), 283-291.

Aristizábal, C. P., Rayo, K. D., Gómez, E. C., y Velázquez, F. A. (2013). La lectura como valor para la construcción del lector competente. Infancias Imágenes, 11(1), 107-113.

Asamblea Médica Mundial (2008). Declaración de Helsinki - Principios éticos para las investigaciones médicas en seres humanos. Corea, Seúl: AMM.

Axelsson, A., Granlund, M., \& Wilder, J. (2013). Engagement in family activities: a quantitative, comparative study of children with profound intellectual and multiple disabilities and children with typical development. Child: care, health and development, 39(4), 523-534.

Ballesteros, J. C., De Asís, J. F., Rodríguez, M. A. y Megías, E. (2009). Ocio (y riesgos) de los jóvenes madrileños. Madrid: Fundación de Ayuda Contra la Drogadicción (FAD).

Berntsson, L. \& Ringsberg, K. (2014). Health and relationships with leisure time activities in Swedish children aged 2-17 years. Scandinavian journal of Caring Sciences, 28(3), 552-563. 
Biemiller, A. \& Boote, C. (2006). An effective method for building meaning vocabulary in primary grades. Journal of Educational Psychology, 98(1), 4462.

Bygren, L. O., Weissglas, G., Wikström, B., Konlaan, B. B., Grjibovski, A., Karlsson, A., Andersson, S., \& Sjöström, M. (2009). Cultural participation and health: a randomized controlled trial among medical care staff. Psychosomatic Medicine, 71(4), 469-473.

Caballo, M. B., Caride, J. A., y Gradaílle, R. (2012). Entre los tiempos escolares y los tiempos de ocio: su incidencia en la vida cotidiana de los adolescentes que cursan la Educación Secundaria obligatoria en España. Educar em Revista, 1(45), 37-55.

Caride, J. A., Lorenzo, J. J. y Rodríguez, M. A. (2012). Educar cotidianamente: el tiempo como escenario pedagógico y social en la adolescencia escolarizada. Pedagogía social. Revista interuniversitaria, 20(1), 19-60.

Cassián, N. (2012). De qué está hecha una ciudad creativa. Una propuesta para abordar la cultura, el ocio y la creatividad en la urbe contemporánea. Athenea Digital. Revista de pensamiento e investigación social, 12(1), 169-190.

Cerrillo, P. C., Yubero, S., y Larrañaga, E. (2004). Valores y lectura. Estudios multidisciplinares. Castilla La Mancha: Universidad de Castilla La Mancha.

Conejo, P. A. (2012). El valor formativo de la música para la educación en valores", DEDICA. Revista de Educación y Humanidades, 2(1), 263-278.

Conlon, E., Zimmer, M., Creed, P., \& Tucker, M. (2006). Family history, selfperceptions, attitudes and cognitive abilities are associated with early adolescent reading skills. Journal of Research in Reading, 29(1), 11-32.

Cromley, J. (2009). Reading, Achievement and Science Proficiency: International Comparisons from the Programme on International Student Assessment. Reading Psychology, 30(2), 89-118.

Cuenca, M. (2000). Ocio Humanista. Dimensiones y manifestaciones actuales del ocio. Bilbao: Universidad de Deusto.

-. (2004). Pedagogía del Ocio: Modelos y Propuestas. Bilbao: Universidad de Deusto.

-. (2009). La Pedagogía del Ocio: Nuevos Desafios. Lugo: Axac.

Cuenca, M. y Goytia, A. (2012). Ocio experiencial: antecedentes y características. Arbor, Ciencia, pensamiento y cultura, 188(754), 265-281.

Cuypers, K., Krokstad, S., Holmen, T., Knudtsen, M., Olov, L., \& Holmen, J. (2012). Patterns of receptive and creative cultural activities and their association with perceived health, anxiety, depression and satisfaction with life among adults: the HUNT study, Norway. Journal of Epidemiology and Community Health, 66(8), 698-703. 
De Vries, J. \& De Graaf, P. (2008). Is the intergenerational transmission of high cultural activities biased by the retrospective measurement of parental high cultural activities? Social Indicators Research, 85(2), 311-327.

Díaz, A. (2009). La importancia de la lectura. Revista de Innovación y Experiencias educativas, 15(1), 1-9.

Elchardus, M. (2009). Self-control as social control: the emergence of symbolic society", Poetics, 37(2), 146-161.

García-Castilla, F.J.; De Juanas Oliva, A.; López-Noguero, F. (2016). La práctica de ocio deportivo de los jóvenes en situación de vulnerabilidad. Revista de Psicología del Deporte, 25(2): 27-32.

Gil, J. (2011). Hábitos lectores y competencias básicas en el alumnado de Educación Secundaria Obligatoria. Educación XXI, 14(1), 117-134.

Gran, I. \& Mads Meier, J. (2015). Cultural capital in context: Heterogeneous returns to cultural capital across schooling environments. Social science research, 50(1), 177-188.

Grossi, E., Blessi, G., Sacco, P. L., \& Buscema, M. (2011). The interaction between culture, health and psychological well-being: Data mining from the Italian culture and well-being project. Journal of Happiness Studies, 13(1), 199-248.

Katz-Gerro, T., Raz, S., \& Yaish, M. (2009). How do class, status, ethnicity, and religiosity shape cultural omnivorousness in Israel? Journal of Cultural Economics, 33(1), 1-17.

López Quintás, A. (2013). El Poder formativo de la música. Revista Española de Pedagogía, 1(254), 49-57.

López Ruiz, J. A. (2011). Los jóvenes españoles y el ocio: Un análisis partiendo del estudio empírico. Miscelánea Comillas, 69(135), 495-520.

Madariaga, A. y Cuenca, J. (2011). Los valores del ocio: cambio, choque e innovación. Bilbao: Documentos de Estudios de Ocio, Universidad de Deusto.

Mads, J. (2009). Equal assess but unequal outcomes: Cultural capital and educational choice in a meritocratic society. Social Forces, 87(4), 1943-1971.

Magaña, M. (2006). ¿Qué es la comunicación? División de educación continua. México: Facultad de Psicología, Universidad Nacional Autónoma de México.

McCammon, L, Saldaña, J., Hines, A., \& Omasta, M. (2012). Lifelong Impact: Adult Perceptions of Their High School Speech and/or Theatre Participation. Youth Theatre Journal, 26(1), 2-25.

Ministerio de Educación, Cultura y Deporte (MECD) (2015). Encuesta de hábitos y prácticas culturales en España 2014-2015: Sintesis de resultados. Madrid: Publicación del Ministerio de Educación, Cultura y Deporte.

Moreno, E. (2002). La familia como institución sociocultural: su papel en la adquisición de los hábitos lectores. Portularia: Revista de trabajo social, 2(1), 309-324. 
- (2013). Análisis de la influencia de la familia en los hábitos lectores de sus hijas e hijos: un estudio etnográfico. Contextos Educativos. Revista de Educación, 4(1), 177-196.

Moreno, I. (2011). Los valores del ocio: cambio, choque e innovación. Bilbao: Universidad de Deusto.

Moreno, A. y Rodríguez, E. (2014). Informe de Juventud en España 2012. Metamorfosis, 1, 112-118.

Muñoz, J. M. y Olmos, S. (2010). Adolescencia, tiempo libre y educación. Un estudio con alumnos de la ESO. Educación XX1, 13(2), 139-162.

Orduna, G. y Urpí, C. (2010). Turismo cultural como experiencia educativa de ocio. Polis 9(26), 85-108.

Piasta, S., Justice, L., McGinty, A., \& Kaderavek, J. (2012). Increasing your children's contact with print during shared reading: Longitudinal effects on literacy achievement. Child Development, 83(3), 810-820.

Pinxten, W. \& Lievens, J. (2014). The importance of economic, social and cultural capital in understanding health inequalities: using a Bourdieu based approach in research on physical and mental health perceptions. Sociology of health \& illness, 36(7), 1095-1110.

Presentado, E., Campuzano, A., Cáceres, G., Cárdenas, A., Fernández, M. C., González, S., y Catalina, S. (2014). Prácticas sobre la lectura de libros a niños y niñas en una comunidad de Paraguay. Pediatría (Asunción), 41(2), 108-112.

Ramírez-Marrero, F., Smith, B., Meléndez-Brau, N., \& Santana-Bagur, J. (2004). Physical and leisure activity, body composition, and life satisfaction in HIVpositive Hispanics in Puerto Rico. Journal of the Association of Nurses in AIDS Care, 15(4), 68-77.

Vanherwegen, D. \& Lievens, J. (2014). The Mechanisms Influencing Active Arts Participation: An Analysis of the Visual Arts, Music, and the Performing Arts. Sociological Inquiry, 84(3), 435-471.

Valdemoros, M. Á., Ponce-de-León, A., Sanz, E., y Tierno, J. (2015). La asistencia a espectáculos en el ocio cultural compartido en familia de los jóvenes riojanos. Berceo. Revista riojana de ciencias sociales y humanidades, 169(29), 191-203.

Veenstra, G. \& Patterson, P. (2012). Capital relations and health: mediating and moderating effects of cultural, economic, and social capitals on mortality in Alameda County, California. International Journal of Health Services, 42(2), 277-291.

Yubero, S. y Larrañaga, E. (2010). El valor de la lectura en relación con el comportamiento lector. Estudio sobre hábitos lectores y estilo de vida en niños. Ocnos, Revista de estudios sobre lectura, 6(1), 7-20.

Zucker, T., Cabell, S., Justice, L., Pentimonti, J., \& Kaderavek, J. (2013). The role of frequent, interactive prekindergarten shared reading in the longitudinal 
development of language and literacy skills. Developmental Psychology, 49(8), 1425-1439.

Eva Sanz Arazuri

Didáctica de la Expresión Corporal

Facultad de Letras y de la Educación Universidad de La Rioja Universidad de La Rioja, Luis de Ulloa 2, 26004 Logroño (España) https://orcid.org/0000-0003-3035-8751

$\mathrm{M}^{\mathrm{a}}$ Ángeles Valdemoros San Emeterio Teoría e Historia de la Educación, Facultad de Letras y de la Educación Universidad de La Rioja Universidad de La Rioja, Luis de Ulloa 2, 26004 Logroño (España) https://orcid.org/0000-0002-7389-4039
Carmen Duque Palacios Subdirección General de Universidades y Formación Permanente

Gobierno de La Rioja https://orcid.org/0000-0002-0444-2995 Francisco Javier García-Castilla Departamento de Trabajo Social Facultad de Derecho UNED https://orcid.org/0000-0003-3040-640X 
